\title{
ASSOCIATION OF PERFORATION PERITONITIS IN EXTRAPERITONEAL SURGERY- AN UNRELATED COMPLICATION
}

\author{
Saleem Tahir', Shariq Ahmad² \\ ${ }^{1}$ Associate Professor, Department of Surgery, Era's Lucknow Medical College and Hospital, Lucknow. \\ ${ }^{2}$ Senior Resident, Department of Surgery, Era's Lucknow Medical College and Hospital, Lucknow.
}

\section{ABSTRACT}

\section{BACKGROUND}

The aim of this study is to discuss and emphasise the occurrence of perforated diverticular disease as an unrelated complication in extraperitoneal surgery.

Objective- 35 patients are reported here where perforation of colonic diverticulum took place in 7 patients after an extraperitoneal surgery and emphasising the unrelated complication.

\section{MATERIALS AND METHODS}

The study comprised of 35 patients of extraperitoneal surgery and the incidence of perforation recorded in total patients.

\section{RESULTS}

Perforation of colonic diverticula was observed in 7 patients who underwent extraperitoneal surgery.

Observation- Perforation of colonic diverticula is multifactorial and the incidence has increased in patients undergoing surgery with multiple factors.

\section{CONCLUSION}

Spontaneous perforation of sigmoid diverticula is multifactorial and the differential diagnosis of an unrelated complication in extraperitoneal surgeries should be kept in mind based on the clinical symptomology.

\section{KEYWORDS}

Perforation Peritonitis in Extraperitoneal Surgery.

HOW TO CITE THIS ARTICLE: Tahir S, Ahmad S. Association of perforation peritonitis in extraperitoneal surgery- an unrelated complication. J. Evolution Med. Dent. Sci. 2017;6(20):1632-1635, DOI: 10.14260/Jemds/2017/357

\section{BACKGROUND}

Diverticular disease has an increasing incidence in western countries, which can be explained by an increasing elderly population and also the relatively low-fibre diet of Western culture. Fifty percent of the population over 50 years of age have diverticulosis, and it is estimated that acute diverticulitis will develop in $15-20 \%$ of these cases.(1) Complicated diverticular disease carries significant morbidity and mortality. These were influenced by patient-related factors. Because of high mortality and morbidities, it is suggested the need to target a specific group of patients for early diagnosis and prophylactic management.

\section{MATERIALS AND METHODS}

The study was conducted in Era's Medical College, Lucknow. It was done on 35 patients over a period of 2 years.

All the patients were above 40 years of age and planned for elective renal surgeries; 14 patients were planned for elective open nephrectomy, 6 of left side and 8 of right side; 21 patients were planned for PCNL, 13 of right side and 8 of left side.

Financial or Other, Competing Interest: None.

Submission 26-01-2017, Peer Review 23-02-2017,

Acceptance 01-03-2017, Published 09-03-2017.

Corresponding Author:

Dr. Saleem Tahir,

Associate Professor

Department of Surgery,

Era's Lucknow Medical College \& Hospital,

Lucknow-226003, Uttar Pradesh, India.

E-mail:dr_tahir75@yahoo.com

DOI: $10.14260 /$ jemds $/ 2017 / 357$

\section{(c) (i) $\$$}

All the routine investigations done preoperatively were within normal limits and the patients were declared fit in preanaesthetic check-up.

\section{RESULTS}

7 of the 35 patients developed symptoms of bowel obstruction in the post-operative period.

The patients developed distension of abdomen and features of peritonitis in the post-operative period from Day 5 to Day 16.

Exploratory laparotomies of all patients revealed sigmoid perforation in 5 patients, perforation of descending colon in 1 patient and caecal perforation in 1 patient.

In 4 of the above patients, there were multiple diverticula in whole of the colon including sigmoid and caecum.

Temporary ileostomy was done in 6 patients and caecostomy was done in 1 patient.

Post laparotomy mortality was found in 1 patient, while others responded well in the post-operative period.

All the other patients of the 35 were discharged uneventfully.

25 patients gave a history of constipation previously, while others did not have any altered bowel and bladder habits.

The association was $71.2 \%$ with respect to all the cases with preop constipation. All the patients who had signs and symptoms of perforation had history of constipation. Colonoscopy was not done preoperatively in any of the patients.

The diagnosis of perforation was made on post-operative chest $\mathrm{x}$-ray suggesting gas under diaphragm and inter-bowel fluid on ultrasonogram. 


\begin{tabular}{|c|c|c|c|c|c|}
\hline & Patient 1 & Patient 2 & Patient 3 & Patient 4 & Patient 5 \\
\hline Age & 46 & 62 & 55 & 65 & 69 \\
\hline Type of surgery & Right PCNL & Left nephrectomy & Right nephrectomy & Right PCNL & Left nephrectomy \\
\hline Post-operative constipation & Present & Absent & Present & Present & Absent \\
\hline $\begin{array}{c}\text { Findings } \\
\text { on exploratory laparotomy }\end{array}$ & Sigmoid perforation & $\begin{array}{c}\text { Descending colon } \\
\text { perforation }\end{array}$ & Sigmoid perforation & $\begin{array}{c}\text { Sigmoid } \\
\text { perforation }\end{array}$ & Caecal perforation \\
\hline \multicolumn{4}{|c|}{ Table 1 } \\
\hline
\end{tabular}

Pre- and Post-operative Blood Values

\begin{tabular}{|c|c|c|c|c|c|c|c|c|c|c|}
\hline & \multicolumn{2}{|c|}{ Patient 1 } & \multicolumn{2}{c|}{ Patient 2 } & \multicolumn{2}{c|}{ Patient 3 } & \multicolumn{2}{c|}{ Patient 4 } & \multicolumn{2}{c|}{ Patient 5 } \\
\hline & Pre & Post & Pre & Post & Pre & Post & Pre & Post & Pre & Post \\
\hline Hb (gm\%) & 11.1 & 10.9 & 12 & 12.2 & 13.1 & 12.6 & 10.8 & 11 & 10.7 & 10.4 \\
\hline TLC (/mm3) & 7300 & 11000 & 9200 & 14300 & 6000 & 7400 & 8200 & 7350 & 12000 & 10900 \\
\hline S. K+ (mEq/L) & 3.8 & 2.7 & 4.1 & 3.4 & 4.4 & 3.6 & 3.5 & 2.8 & 4.3 & 3.9 \\
\hline S. Bilirubin (mg\%) & 0.4 & 0.6 & 0.9 & 1.1 & 0.9 & 0.7 & 1.1 & 1 & 0.3 & 0.6 \\
\hline
\end{tabular}

The preoperative and postoperative haemoglobin levels were within normal limits, but there was found to be a decrease in serum potassium levels in the post-operative period indicating that post-operative ileus may have caused the rupture of the diverticula spontaneously. The pre- and post-operative TLC and serum bilirubin levels were insignificant. But the TLC increased when there were features of peritonitis from POD 6 to POD 16. In all the above cases during the extraperitoneal first surgery, care was taken not to breach the peritoneum and no iatrogenic bowel injury was registered. Respiratory complications and long-term steroid use was ruled out in all of these patients except one who had a history of bronchial asthma and was on bronchodilators for 10 years.

\section{Association of Peritonitis and Renal Surgeries}

\begin{tabular}{|c|c|c|}
\hline Observation & Nephrectomy & PCNL \\
\hline Total cases with perforation & 5 & 2 \\
\hline Postoperative constipation & 18 & 7 \\
\hline Sigmoid perforation & 3 & 2 \\
\hline Age $>60$ years & 7 & 11 \\
\hline Use of bronchodilators & 1 & none \\
\hline
\end{tabular}

Percentage of patients who underwent nephrectomy developing peritonitis $35.70 \%$. Percentage of patients who underwent PCNL developing peritonitis $5.80 \%$. The association of symptoms of peritonitis with nephrectomy was significant unrelated to the side of the surgery. Age had an association of $70 \%$ with development of peritonitis with age being $>60$ years. There was also significant association of constipation in terms of development of peritonitis. In all the patients developing peritonitis history of on and off constipation with irregular bowel habits was present. None of the patients were found to be using corticosteroids for the long time with no history of bronchial asthma and any other respiratory illness.

\section{Diverticular Perforation}

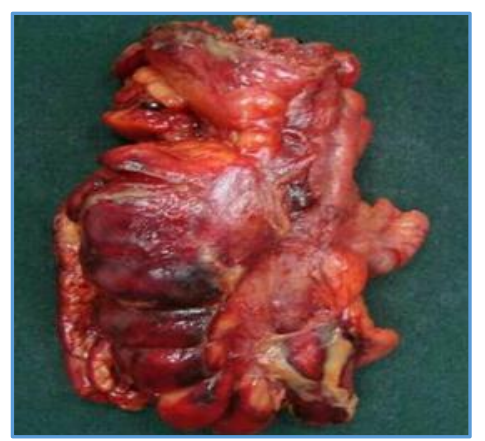

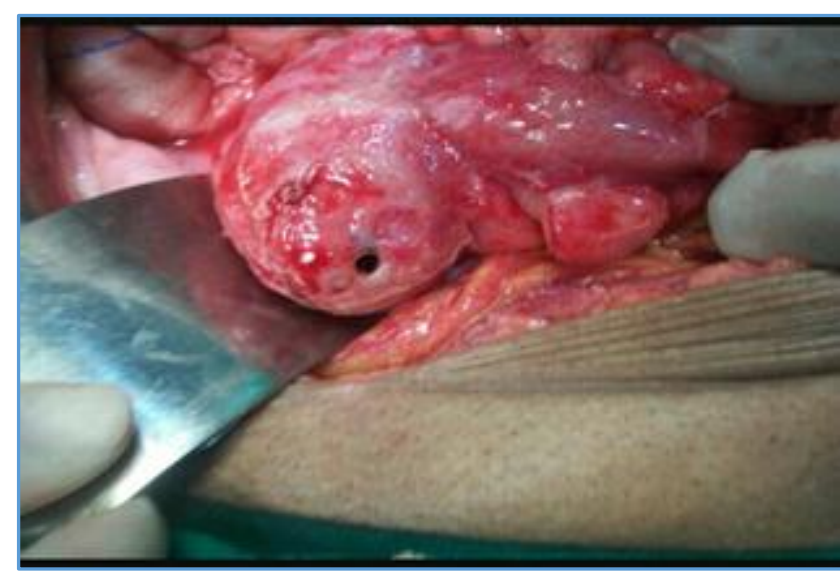

\section{DISCUSSION}

Acute postoperative perforated diverticulitis has been associated with cardiac surgery or renal transplant surgery.(2) The pathogenesis of postoperative diverticulitis is multifactorial in nature. Its high incidence may be common in older age group patients undergoing surgery. Low-fibre diets may also have a role in the pathogenesis of perforated diverticulitis, as they can lead to diverticulosis and chronic constipation. In the postoperative period, there may be worsening constipation due to bed rest, opiate analgesics, anaesthetics and the surgery itself. Another theory in the pathogenesis of diverticular perforation suggests that it is due to intestinal mucosal ischaemia induced by hypotension, low-flow states, the use of vasoconstricting drugs, and microthrombi or emboli.(3) Increased collagenase activity after surgery with resulting collagen breakdown in thinwalled diverticula, is suggested to be a contributing factor to postoperative perforation. ${ }^{(4)}$

The clinical presentation of diverticulitis depends on the location of the affected diverticulum, the severity of the inflammatory process and the presence of complications.

\section{Presenting Complaints include the following-}

- Left lower quadrant pain (70\% of patients).

- Change in bowel habits.

- Nausea and vomiting.

- Constipation.

- Diarrhoea.

- Flatulence.

- Bloating. 
Physical findings in patients with diverticulitis mirror the severity of the inflammation and the presence of complications as follows.

In simple diverticulitis, localised abdominal tenderness in the area of the affected diverticula and fever.

Left lower quadrant tenderness is most common, as most diverticula occur in the sigmoid colon.

Right lower quadrant tenderness, mimicking acute appendicitis can occur in right-sided diverticulitis.

In complicated diverticulitis with abscess formation a tender palpable mass, elderly patients and some patients taking corticosteroids may have unremarkable findings, even in the presence of severe diverticulitis, findings in patients with peritonitis due to free perforation are as follows.

Generalised tenderness with rebound and guarding on abdominal examination, the abdomen may be distended and tympanic to percussion, bowel sounds can be diminished or absent, the fibre content of the diet plays a large role in the pathogenesis of diverticular disease. Fibre has been found to be protective. Most fibre in the human diet is of plant origin and this type of fibre binds water and salt in the colon, leading to bulkier and more voluminous stools. Therefore, fibre decreases the frequency of contractions and prevents an exaggerated form of segmentation. In addition, dietary fibre influences the content of colonic bacterial flora, forms the main substrate for bacterial carbohydrate fermentation and produces energy-yielding substrates-short-chain fatty acids-for growth and maintenance of colonic cellular function. Consequently, a fibre-deficient diet increases the chances of intense, more frequent segmentation, thus predisposing to herniation of mucosa by allowing isolated increases of intraluminal pressure.

\section{Summary}

The case series includes 35 patients of extraperitoneal surgery including PCNL and nephrectomy. Perforation peritonitis was observed in 5 patients that underwent surgery. Laparotomy in all of these patients revealed ruptured colonic diverticula. In all of these patients, there was no significant association with any of the risk factors and hence the need for preoperative colonoscopy was unwarranted. On close history taking of the previous incidence revealed episodes of altered bowel habits in some of the patients. However, postoperative constipation, use of asthmatic drugs and associated comorbidities played important factors. Henceforth, the important points from this study revealed that bowel pathology, especially colonic diverticula may be considered in all cases of extraperitoneal surgeries if the patient presents with post-operative abdominal distension and features of peritonitis more so if the patient is associated with certain risk factors. The management in these patients should be aimed at early diagnosis and aggressive management, which includes either a diversion procedure or resection and anastomosis. Followup of these patients must be regularly done with colonoscopy and maintenance of a high-fibre diet with reduction of episodes of constipation. The use of corticosteroids and opioids must be under strict control of the physician. Most cases detected early can be treated conservatively and on bowel rest. Surgical management is done in severe cases or patients with peritonitis. The differential diagnosis involves carcinoma colon, inflammatory bowel disease and irritable bowel syndrome. The main aim is avoidance of complication, mainly stricture and perforation. The prognosis if detected early is favourable and mortality is low.

\section{CONCLUSION}

Spontaneous perforation of sigmoid diverticula is multifactorial and the differential diagnosis of an unrelated complication in extraperitoneal surgeries should be kept in mind based on the clinical symptomology. The perforation of colonic diverticula has been observed previously in surgeries such as cardiac bypass and renal transplantation, but perforation in routine elective surgeries warrant a keen history taking and thorough investigation in all suspected patients planned for even extraperitoneal elective surgeries. Perforation has been linked to Non-Steroidal AntiInflammatory Drug (NSAID) use in case-control studies. Glucocorticoids may increase this risk. Steroids also may mask symptoms and delay appropriate therapy. Because of this, NSAIDs and glucocorticoids should be used with caution in patients who have known diverticular disease or in patients who are at risk. Diffuse purulent or faecal peritonitis is the most dramatic complication, which still has a high risk of mortality and morbidity. Surgical risk is related to clinical conditions, duration of peritonitis, age of patient and comorbidities. Thus, it is not possible to state a univocal approach, although Hartmann's procedure keeps being the first choice.(5) But still the treatment of primary resection with or without a diversion colostomy is debatable. The initial assessment of patients with suspected acute diverticulitis is comprised of a thorough history and physical examination including abdominal, rectal and pelvic examinations. Useful initial examinations may include a complete blood cell count, urinalysis and flat and upright abdominal radiography. If the clinical picture is clear enough to diagnose diverticulitis, no other tests are indicated. For confirmation other tests such as Computed Tomography (CT), water-soluble contrast enema, endoscopy and ultrasound may be performed. In particular, the differential diagnosis of colorectal cancer should also be kept in mind. A barium enema examination should be avoided in acute presentations in patients with suspected acute diverticulitis and localised peritoneal signs because of a possible extravasation of barium into the peritoneal cavity, which can increase the morbidity and mortality related to barium-induced chemical peritonitis. In the emergency setting, water-soluble enemas are safer. There are several important points regarding surgical technique. From a technical standpoint, the resection should be carried proximally to the compliant bowel and extend distally to the upper rectum. After sigmoid colectomy, an important predictor of recurrence is a colosigmoid rather than colorectal anastomosis. The proximal margin of resection should be in an area of pliable colon without hypertrophy or inflammation. Resection of the diseased colon must be the desired goal along with removal of the entire thickened colonic segment(s), but not necessarily all the proximal diverticula-bearing colon. Laparoscopic colectomy is appropriate in select patients and has advantages over open laparotomy including less pain, smaller incisions and shorter recovery. There is no increase in early and late complications and cost and outcome are comparable with those of open resection. 


\section{REFERENCES}

[1] Hackford AW, Veidenheimer MC. Diverticular disease of the colon. Surg Clin N Am 1995;65:347-63.

[2] Soravia C, Baldi A, Kartheuser A, et al. Acute colonic complications after kidney transplantation. Acta Chir Belg 1995;95(3):157-61.

[3] Mirvis S, Scovill WA. Colonic diverticulum perforation: report of two cases as a complication of CABG. Am J Gastroenterol 1985;80(7):547-9.
[4] Voitk AJ, Mustard RA. Perforated diverticulitis after surgery. Can J Surg 1989;32(5):370-2.

[5] Tonelli F, Di Carlo V, Liscia G, et al. Diverticular disease of the colon: diagnosis and treatment. Consensus conference, $5^{\text {th }}$ national congress of the Italian society of academic surgeons. Ann Ital Chir 2009;80(1):3-8. 